\title{
ORIGINAL ARTICLE Modulation of cue-induced firing of ventral tegmental area dopamine neurons by leptin and ghrelin
}

\author{
G van der Plasse ${ }^{1,4}, \mathrm{R}$ van Zessen ${ }^{1,4}$, MCM Luijendijk $^{1}, \mathrm{H}$ Erkan $^{1,2}, \mathrm{GD}$ Stuber $^{3}, \mathrm{GMJ}$ Ramakers ${ }^{1}$ and RAH Adan $^{1}$
}

BACKGROUND/OBJECTIVES: The rewarding value of palatable foods contributes to overconsumption, even in satiated subjects. Midbrain dopaminergic activity in response to reward-predicting environmental stimuli drives reward-seeking and motivated behavior for food rewards. This mesolimbic dopamine (DA) system is sensitive to changes in energy balance, yet it has thus far not been established whether reward signaling of DA neurons in vivo is under control of hormones that signal appetite and energy balance such as ghrelin and leptin.

SUBJECTS/METHODS: We trained rats $(n=11)$ on an operant task in which they could earn two different food rewards. We then implanted recording electrodes in the ventral tegmental area (VTA), and recorded from DA neurons during behavior. Subsequently, we assessed the effects of mild food restriction and pretreatment with the adipose tissue-derived anorexigenic hormone leptin or the orexigenic hormone ghrelin on VTA DA reward signaling.

RESULTS: Animals showed an increase in performance following mild food restriction $(P=0.002)$. Importantly, food-cue induced DA firing increased when animals were food restricted $(P=0.02)$, but was significantly attenuated after leptin pretreatment $(P=0.00)$. While ghrelin did affect baseline DA activity $(P=0.025)$, it did not affect cue-induced firing $(P \geqslant 0.353)$.

CONCLUSIONS: Metabolic signals, such as leptin, affect food seeking, a process that is dependent on the formation of cue-reward outcomes and involves midbrain DA signaling. These data show that food restriction engages the encoding of food cues by VTA DA neurons at a millisecond level and leptin suppresses this activity. This suggests that leptin is a key in linking metabolic information to reward signaling.

International Journal of Obesity (2015) 39, 1742-1749; doi:10.1038/ijo.2015.131

\section{INTRODUCTION}

The worldwide prevalence of obesity and the ongoing debate with respect to the existence of eating-addiction illustrates the importance of a precise understanding of the neurobiology of feeding behavior. ${ }^{1}$ The maintenance of a positive energy balance is regulated by multiple neural circuits that control energy expenditure and the procurement of energy sources. In addition to metabolic centers located in the hypothalamus that sense and regulate energy homeostasis, ${ }^{2}$ dopaminergic (DA) neurons in the midbrain (ventral tegmental area (VTA) and substantia nigra) have a crucial role in reinforcing food seeking behavior., ${ }^{3,4}$

Activity of VTA DA neurons that project to the ventral striatum, which is important for feeding behavior, ${ }^{5,6}$ is necessary for the formation of cue-reward associations and effort-related food seeking. ${ }^{7}$ Previous experiments in monkeys show that these DA neurons have an important role in signaling the value of foodpredicting cues that drive motivated behavior. ${ }^{8}$ Importantly, this DA signal also encodes properties like reward identity and size, which allow for an accurate behavioral response following food availability. $^{9-12}$

Of particular interest for feeding behavior is that the activity of the midbrain DA system is modulated by metabolic state and feeding hormones. ${ }^{13-15}$ As such, under normal physiological conditions, the DA system can drive food-seeking behavior during hunger, and reduce this activity when satiated. Chronic food restriction, for example, reduces the levels of the anorexic hormone leptin levels ${ }^{16}$ and increases overall DA neuronal burst firing. ${ }^{17,18}$ In contrast, suppression of dopaminergic activity is observed following administration of leptin in anesthetized rats. ${ }^{19}$ In direct opposition to leptin, the orexigenic hormone ghrelin increases DA release in vivo. ${ }^{20}$ Despite the correlation between feeding hormones, DA activity and metabolic state, the functional consequences of leptin and ghrelin on DA neuronal activity during behavior have not been established.

Previously reported effects observed after administration of these hormones show respectively decreased-, and increased motivation to obtain food reward. ${ }^{21,22}$ Of particular relevance here are findings that show that leptin-deficient people show increased cue reactivity in DA-responsive brain regions such as the ventral striatum, even in conditions of satiation, and that leptin normalizes this activity. ${ }^{23}$ Similarly, several studies in human subjects indicate that leptin treatment of congenital-, and acquired leptin deficiency alters brain activity of reward-related brain areas such as the striatum and midbrain. ${ }^{23-25}$ Likewise, functional magnetic resonance imaging data show ghrelin to increase activity of areas that control appetitive behavior ${ }^{26}$ such as the mesolimbic system. ${ }^{27}$

These data suggest that leptin and ghrelin can modulate food seeking and activity of the reward circuitry. However, these studies

${ }^{1}$ Department of Translational Neuroscience, Brain Center Rudolf Magnus, University Medical Center Utrecht, Utrecht, The Netherlands; ${ }^{2}$ Master's Programme Neuroscience and Cognition, Utrecht University, Utrecht, The Netherlands and ${ }^{3}$ Department of Psychiatry \& Cell Biology and Physiology, UNC Neuroscience Center, University of North Carolina at Chapel Hill, Chapel Hill, NC, USA. Correspondence: Dr G van der Plasse, Department of Translational Neuroscience, Brain Center Rudolf Magnus, Universiteitsweg 100, Utrecht 3584 CG, The Netherlands.

E-mail: g.vanderplasse@umcutrecht.nl

${ }^{4}$ These authors contributed equally to this work.

Received 18 February 2015; revised 8 June 2015; accepted 12 July 2015; accepted article preview online 17 July 2015 ; advance online publication, 11 August 2015 
do not identify the neuronal substrate through which these hormones act on the brain to alter cue responsivity.

Although leptin and ghrelin affect food seeking, it is unclear whether these hormones act directly on the signaling of rewardpredicting stimuli. In this study, we determine the ability of ghrelin and leptin to affect the DA reward-system during reward-seeking behavior. These experiments show that a mild food restriction increases the activity of DA neurons in response to a rewardpredicting cue, and pretreatment with leptin abolishes this activity. These data identify leptin as a key hormonal signal in the regulation of DA activity and establish a mechanism through which leptin signaling can modulate food seeking behavior.

\section{MATERIALS AND METHODS}

All experiments were approved by the Animal Experimentation Committee of the University Utrecht and were carried out in agreement with Dutch Law (Wet op de Dierproeven, 1996) and European regulations (Guideline $86 / 609 /$ EEC).

\section{Animals}

Data were collected from 11 male Wistar rats (Harlan CPB, Horst, The Netherlands) weighing on average $391 \pm 12 \mathrm{~g}$ at the time of surgery. On arrival, the animals were individually housed, weighed and handled daily, and kept under a reversed day/night cycle (10:00-22:00 h lights off). Food and water were ad libitum available in the home-cage throughout the experiment.

\section{Apparatus}

Animals were housed and measured in Perspex test chambers $(50 \times 50 \times 35 \mathrm{~cm})$ equipped with a house light, two trial lights and sound cues, three nosepoke units and three food-dispensers (Med Associates, Sandown Scientific, Middlesex, UK). Entries into the nosepoke unit and delivery of food pellets into the chamber were recorded through infra-red detection. Cues and feeders were controlled through a Med-pc interface that also registered all events. Additional event registration was achieved through coupling to the electrophysiology setup (see below).

\section{Surgery}

Surgical procedures for the placement of recording electrodes were identical to those described earlier. ${ }^{28}$ In brief, after induction of anesthesia and exposure of the cranium, a hole was drilled over the VTA in the right hemisphere $\left(5.6 \mathrm{~mm}\right.$ posterior, $1.3 \mathrm{~mm}$ lateral to bregma) ${ }^{29}$ The dura was opened and electrodes were lowered into the brain under a $5^{\circ}$ angle. Sixteen electrodes were bundled into four tetrodes that were mounted in a Harlan 4 Drive (Neuralynx, Bozeman, MT, USA). After lowering the tetrodes $\sim 4 \mathrm{~mm}$ into the brain the drive was anchored to skull-screws with dental cement. Following surgery, tetrodes were further lowered into the target area $(\sim 8.2 \mathrm{~mm} \mathrm{DV})$. Subsequently, the animals were given analgesics for 2 days, and at least 7 days of recovery before electrophysiological recordings started.

\section{Recording}

Recordings were performed in a test chamber that was identical to the home-cage. Data were recorded at $40 \mathrm{Khz}$ using a Multichannel Acquisition Processor (MAP) recording system (Plexon, Dallas, TX, USA), as described earlier. ${ }^{28}$ A $750-\mu s$ data sample was stored whenever the signal crossed a preset voltage threshold. Data were clustered and analyzed offline (see below).

\section{Behavioral procedure}

Behavioral task. After arrival, animals were trained daily in their home cage on a fixed-ratio 1 task $(1 \mathrm{~h})$. During this behavioral task, a single nosepoke led to the delivery of a foodpellet. After animals learned to associate the nosepoke with a food reward, they were trained on the main paradigm. Task onset was signaled by a houselight, which remained illuminated for the duration of the test session (1 h). Following a 30- to 45-s intertrial interval, trial onset was signaled by onset of the light in the nosepoke hole and a sound cue (Figure 1b). Each reward type (see below) was presented in a pseudo-random manner and was signaled by a unique tone and light cue (Figures $1 \mathrm{~b}$ and $\mathrm{c}$ ). To obtain the reward, animals had to nosepoke at one of two reward sites (corresponding to the available reward type). The first nosepoke after a 5-s interval led to the onset of a feeder and delivery of the food reward. The sound of this feeder onset served as a proximal sound cue, and was used for most of the electrophysiological analyses. Food delivery was approximately $450 \mathrm{~ms}$ after feeder activation, followed by the cessation of the sound/light cue a

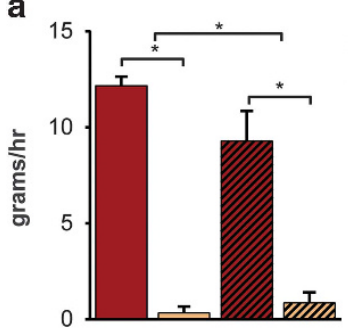

C

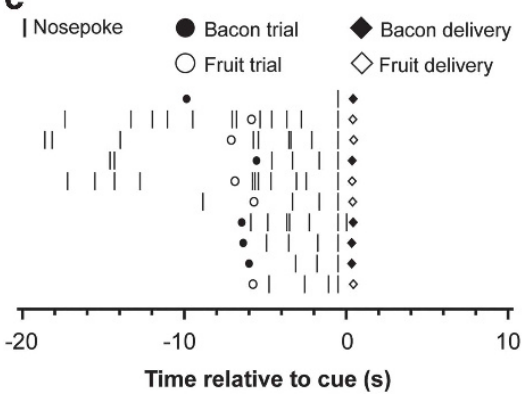

b

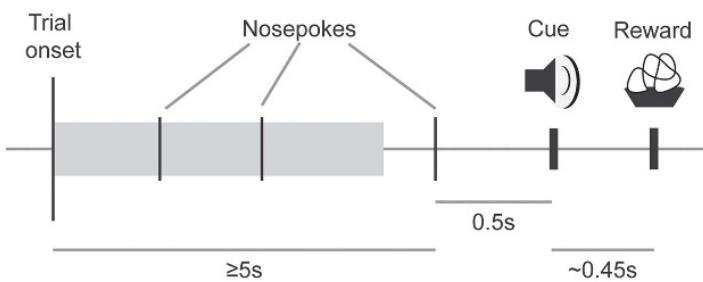

d

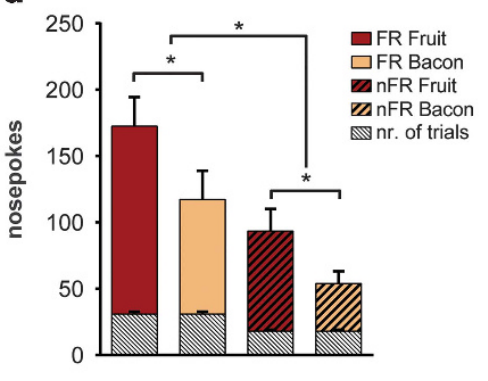

Figure 1. Reward preference, task design and performance. (a) Total intake (g) of fruit- and bacon-flavored rewards during 1-hour free-access in food restricted (FR) and non-restricted (nFR) animals. Data indicate preference for fruit over bacon pellets and increased intake following food restriction. (b) Flowchart of the behavioral task. At trial onset a tone signals the availability of a bacon or fruit reward. The first nosepoke following a 5 -s interval triggers a sound cue $(0.5 \mathrm{~s}$ following the nosepoke) and reward delivery ( $0.45 \mathrm{~s}$ later). (c) Nosepoke activity of a single animal during the first 10 trials of a single session. Rasters are zeroed on the cue. (d) Nosepokes during task execution. Significantly more nosepokes are made for fruit- than for bacon-flavored pellets, food restriction furthermore increases total number of nosepokes. The average number of trials per reward for each session type is depicted in gray. ${ }^{*}$ Significant difference at $P<0.05$. All data are presented as mean \pm s.e.m. 
presentation and trial termination. After completion of the trial, the intertrial interval was reset. During daily training sessions, water and food were freely available. To assess the effect of reward preference on task execution and reward processing, two rewards that were differentially preferred were used. These rewards were bacon-flavored and fruit-flavored $190 \mathrm{mg}$ isocaloric pellets (resp. 3.37 and $3.45 \mathrm{kcal} \mathrm{g}^{-1}$; Bio Serv). 'Bacon' and 'fruit' trials were presented in a pseudo-random order to prevent specific anticipatory activity prior to stimulus presentation (4-trial blocks).

Food preference task. Reward preference was assessed in the recording chamber on separate test days and all experimental conditions were tested (vehicle, hormone and food restriction). Unlimited access to both rewards was given for a period of $1 \mathrm{~h}$. Before and after the session, the total amount of food was weighed and intake was calculated.

Experimental procedure. At the start of an experimental day ( 11:30 h; $1.5 \mathrm{~h}$ into the dark phase), an animal was placed in the recording chamber with either ad libitum or no access to food (food restriction). During this period, ad-lib fed rats have an average intake of $3.34 \pm 1.6 \mathrm{~g}$. Two hours later, the animal was injected intraperitoneal (i.p.) with hormone (leptin or ghrelin) or vehicle (saline) and connected to the recording device. After a 5-min interval, the behavioral task and the electrophysiological recording started. Following task execution, the animals remained in the recording chamber to record baseline neuronal activity (30 min), and neuronal activity following administration of apomorphine (i.p.). To ensure that new neurons were recorded in each condition, the tetrodes were lowered at least $40 \mu \mathrm{m}$ between sessions. Animals were pseudo-randomly chosen for each recording session such that no animal was measured on two consecutive days.

Pharmacological intervention. Ghrelin $\left(250 \mathrm{\mu g} \mathrm{kg}^{-1}\right.$; Tocris, Bristol, UK), leptin ( $1 \mathrm{mg} / \mathrm{kg}$; National Hormone and Peptide Program (NHPP), USA) as well as vehicle (saline) were injected i.p. $5 \mathrm{~min}$ before recordings started. These concentration were previously shown to affect food intake and neuronal activity. ${ }^{28}$ Saline was injected in an identical volume $(0.1 \mathrm{ml}$ per $100 \mathrm{~g}$ ) to control for neuronal responses to the injection. Apomorphine ( $0.1 \mathrm{mg} \mathrm{kg}^{-1}$; Sigma-Aldrich, Saint Louis, MO, USA) was administered i.p. 30 min following task execution, to identify DA neurons.

Data analysis. All statistical analyses were performed using SPSS for Windows (IBM; Armonk, NY, USA) unless stated otherwise. Equality of variance of the differences between groups was assessed using Mauchly's test of sphericity. In case variances were not equal, the degrees of freedom were adjusted by means of a Huyn-Feldt correction to reduce the chance of type-one errors.

Behavior. For the behavioral performance during recording sessions the following measures were analyzed; total number of trials and total number of nosepokes per reward and response latency. During the free-access preference test, the total consumption of each reward was measured.

Electrophysiology. Single neurons were isolated by offline cluster procedures (Offline Sorter x64 V3; Plexon). Cells with a baseline firing frequency of less than $0.1 \mathrm{~Hz}$ over the whole recording were not considered for analysis (identical to Van der Plasse et al. $^{28}$ ). Neurons were classified as DA neurons based on their response to the dopamine 2 receptor agonist apomorphine $(\geqslant 20 \%$ reduction in firing frequency with respect to pre-treatment activity). ${ }^{30}$

To exclude effects of the injection procedure on neuronal activity, data from $30 \mathrm{~s}$ before and until $30 \mathrm{~s}$ after injections were not included in the analyses. Treatment-induced changes in baseline firing frequency were analyzed with a one-way ANOVA and post hoc $t$-test. Burst activity was quantified by computing the fraction of total spikes that occurred within a burst, in accordance with earlier used burst criteria. ${ }^{30,31}$

Event-related firing. Population analyses of neuronal activity were performed to assess neuronal response to the task-related events, cue light (that is, trial onset and type identifier), reward cue (that is, feeder sound), and reward presentation. Reward presentation was measured by an infrared detector placed to detect food pellets at the moment they fell into the cage.

For each single unit, peri-event time histograms were constructed (Neuroexplorer V4, NEX Technologies, Madison, AL, USA) per event Subsequently, binned data $(100 \mathrm{~ms})$ were exported and normalized to baseline using Z-scores based on average firing activity during $10 \mathrm{~s}$ of activity in the intertrial interval. Averaged responses to events were then calculated per condition (hormone/restriction) and neuron type (DA/ other). Statistical analyses were performed by means of a repeatedmeasures ANOVA and post hoc $t$-tests.

\section{Histology}

After the last recording session, rats were anesthetized and the final position of the tetrodes was marked by passing a 10-s, 25- $\mu \mathrm{A}$ current through each tetrode. After $24 \mathrm{~h}$, the animals were killed, and the brains were removed and stored overnight in paraformaldehyde. Subsequently, brains were transferred to a $30 \%$ sucrose solution with $0.05 \%$ sodium azide. Brain sections $(40 \mu \mathrm{m})$ were cut using a vibratome and Nissl stained to identify the location of the final position of the tetrodes.

\section{RESULTS}

Task performance and free choice access reflects reward preference

Animals were trained on a behavioral task in which two different food rewards (fruit- and bacon flavored pellets) could be obtained following a nosepoke response (Figures $1 \mathrm{~b}$ and $\mathrm{c}$ ). During a 1-h free access to both reward types all animals showed preference for fruit-flavored pellets over bacon-flavored rewards (Figure 1a, $t \geqslant 5.065, P=0.000$ ). Furthermore, multivariate analysis of the effects of food restriction on nosepokes per reward type showed a significant effect $(F(2,14)=5,573, P=0.017)$. Subsequent analysis showed that the number of nosepokes for fruit-flavored rewards was significantly greater than for bacon-flavored rewards under conditions of both food restriction and non-food restriction (resp. $F(1,15)=6,369, P=0.023 ; F(1,15)=7,781, P=0.014$; Figure 1d).

Although food restriction in these animals significantly increased overall intake during the $1-\mathrm{h}$ free access $(t=1.839$, $P=0.046$ ), it did not affect preference (Figure 1a). Increased intake following restriction was also reflected in behavioral performance. Animals made significantly more nosepokes under conditions of food restriction than when food was freely available (Figure 1d; $t=3.377, P=0.002$ ). In addition, food restriction (that is, $2 \mathrm{~h}$ of restriction) significantly increased the number of trials that animals performed $(30.8 \pm 1.8)$ compared with free-fed animals $(18 \pm 1.1$ trials; $t=4.392, P=0.001$; Figure $1 \mathrm{~d}$, gray bars). Under ad lib conditions, when food was available during testing, no chow was consumed. Pre-treatment with ghrelin or leptin did not affect the number or delay of nosepokes compared with saline control (resp. $F=0.812, P=0.462$ and $F=0.106, P=0.900$ ), nor reward preference $(F=1.597, P=0.243)$, allowing for the analysis of reward processing independent of task execution.

These data thus indicate that rats show clear reward preference and increased reward-seeking behavior after mild food restriction. Moreover, under these conditions leptin nor ghrelin affected task performance.

Measurement of midbrain neuronal activity revealed neuron typedependent firing in response to cue presentation

To investigate the effect of short food restriction (2h) and hormone treatment on reward processing of midbrain neurons, we recorded single-cell neuronal activity in the VTA of rats $(n=11)$ performing an operant task to receive two different food rewards. Before performing this task, animals were pretreated with ghrelin, leptin or vehicle (saline). During these experiments, 283 midbrain neurons were measured (Figures 2a and b). Of these, 99 were classified as putative DA (Figure $2 \mathrm{~b}$, inner circle) based on their inhibition by the dopamine 2 receptor agonist apomorphine.To rule out effects of apomorphine on task performance, we administered the hormone $30 \mathrm{~min}$ after the end of the behavioral task. The remaining neuronal population was uniformly categorized as non-DA. Figure $2 \mathrm{~b}$ shows a complete overview of the number of neurons measured, per condition. 
a

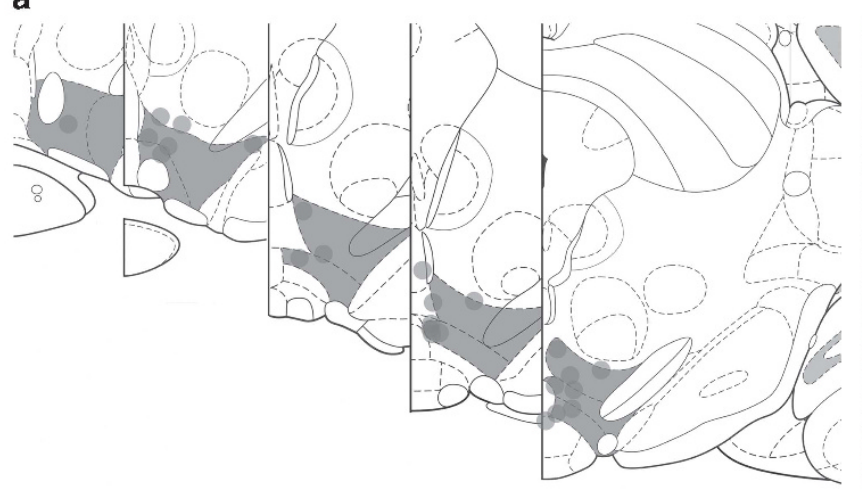

$-5.04 \quad-5.28 \quad-5.52$

b

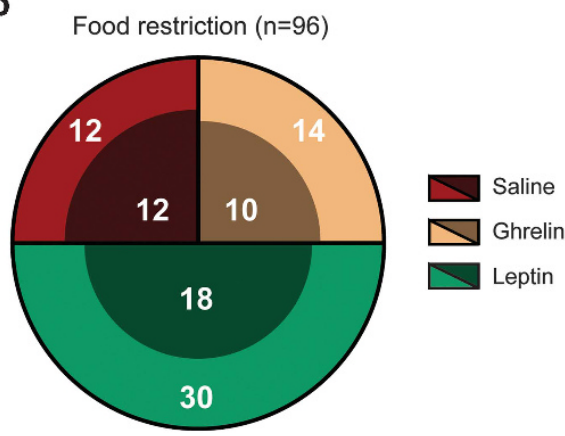

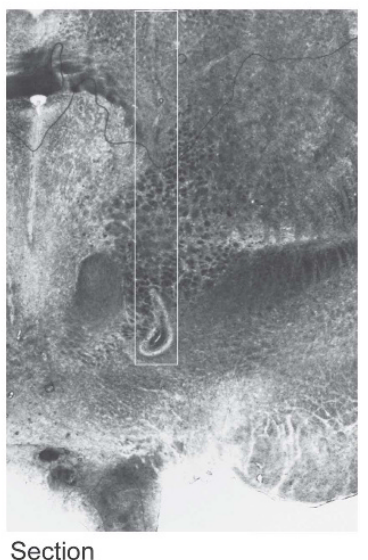

Section

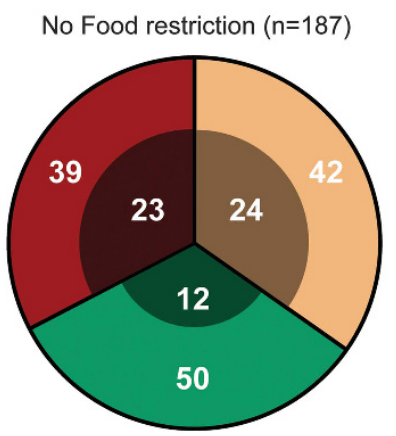

Figure 2. (a) Recording sites in the midbrain. (left) Shaded areas delineate the ventral tegmental area (VTA), tetrode end points are marked as circles. Relative distance to bregma is indicated below. (right) Photograph of a histological section showing a tetrode track (outlined in white) and end point. (b) Overview of the number of recorded neurons per session and condition. Inner circles show dopamine (DA) neurons and non-DA neurons are shown in the outer circle.

Table 1. Baseline firing frequencies and burst activity per condition

\begin{tabular}{lccllc}
\hline & \multicolumn{2}{c}{ No food restriction } & & \multicolumn{2}{c}{ Food restriction } \\
\cline { 2 - 3 } \cline { 6 - 6 } & Firing freq. & SWB & & Firing freq. & SWB \\
\hline Saline & $1.23(0.41)$ & $0.21(0.06)$ & & $1.03(0.26)$ & $0.23(0.04)$ \\
Leptin & $0.73(0.27)$ & $0.19(0.09)$ & & $0.44(0.1)^{*}$ & $0.32(0.06)$ \\
Ghrelin & $1.58(0.87)$ & $0.21(0.06)$ & & $0.31(0.24)^{*}$ & $0.08(0.2)^{* *}$ \\
\hline
\end{tabular}

Overview of average firing frequency ( \pm s.e.m.), and the fraction of spikes that occur within a burst (SWB; \pm s.e.m.) of putative dopamine (DA) neurons. *Significant difference $P<0.05$ and ${ }^{*}$ Significant difference $P<0.01$.

We first assessed the effects of hormone treatment and food restriction on baseline firing frequency (Table 1). ANOVA analyses revealed that hormone treatment affected firing activity under conditions of food restriction $(F=5.100, P=0.011)$, but not when animals were fed ad lib ( $F=0.328, P=0.722)$. In line with previous findings from anesthetized animals, ${ }^{19}$ subsequent post hoc analyses showed that leptin reduces firing frequency of DA neurons in awake rats, during food restriction ( $F=5.761, P=0.023)$. Unexpectedly, a similar reduction of hormone-induced neuronal activity was found for ghrelin under these conditions $(F=5.862$, $P=0.025$; see Abizaid et al. ${ }^{32}$ ). Burst-firing analysis of DA neurons revealed that under saline conditions there was no difference in the fraction of spikes that occur within a burst between restriction and non-food restriction $(21-23 \%$, burst percentage is similar to earlier reports $\left.{ }^{30,31}\right)$. Under conditions of food restriction, burst firing was significantly decreased following ghrelin pre-treatment
( $F=8.833, P=0.008)$ and unaffected by leptin. Thus, both ghrelin and leptin decrease the baseline firing frequency of DA neurons, but only during a mild food restriction.

In accordance with the known neurophysiological properties of midbrain DA neurons, under conditions of a mild food restriction DA neurons showed clear increases in activity in response to a cue presentation that predicted the upcoming reward. Whereas these neurons showed a clear cue-induced increase in firing activity, non-DA neurons showed no such response (Figure 2a). A repeated-measures ANOVA comparison between cue-induced firing in DA and non-DA neurons revealed a significant effect of both time $(F=4.412, P=0.001)$, and group $\times$ time $(F=3.125$, $P=0.009$ ). Figure 3 a shows the average cue-induced changes for both cell types ( $t$-test; 3.524, $P=0.001$ ).

Also, analysis of the whole population revealed a significant increase in neuronal activity following cue onset with respect to baseline ( $r m A N O V A, F=4.039, P=0.004$; simple-first contrast; $\mathrm{F}=5.562, P=0.02$ ). Furthermore, actual reward delivery did not induce differential firing in DA and non-DA neurons $(F=0.313$, $P=0.582$ ), indicating that activity in DA neurons is not attributable to the sensory processing of the actual reward.

As DA neurons have previously been shown to encode relative reward value, and in these experiments rats showed preference for fruit-flavored rewards, we assessed whether DA neurons exhibit differential firing in response to cue and reward presentation. In an unbiased approach we compared the first $500 \mathrm{~ms}$ after cue onset, for all neurons recorded under saline conditions. We found differential neuronal activity between the two rewards $300-400 \mathrm{~ms}$ after cue onset with increased activity for the preferred fruit reward, similar to what has been reported. ${ }^{33}$ Importantly, this difference was significant only in the population 


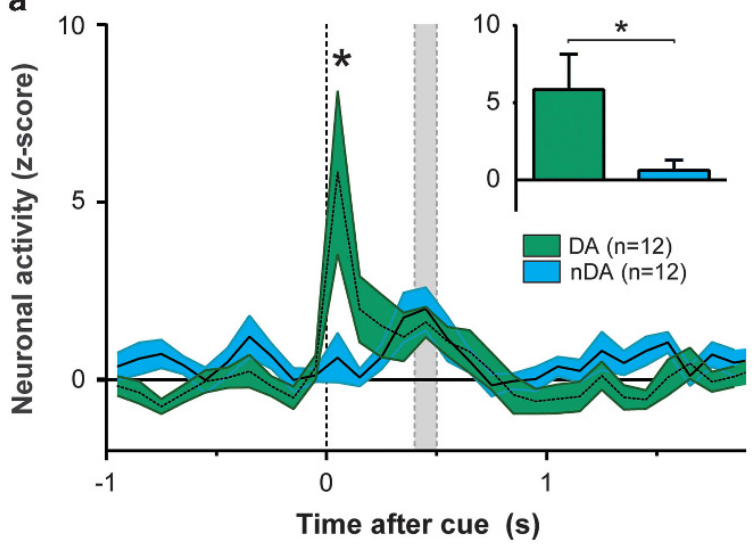

b

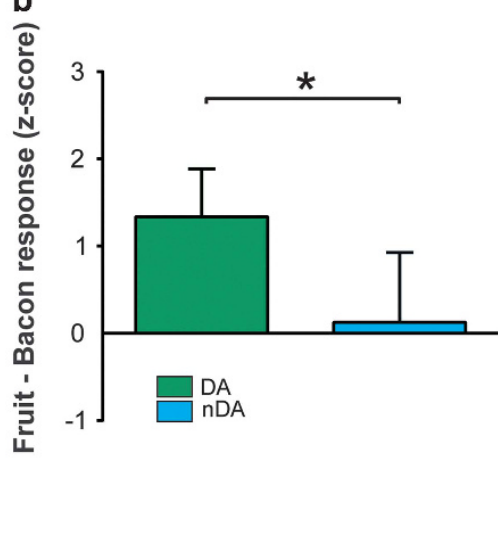

Figure 3. Electrophysiological recordings in the rat midbrain. (a) Significant cue-induced neuronal firing in dopamine (DA)-, but not in non-DA (nDA) neurons under food-restriction conditions (resp. green and blue trace). The insert shows a significant difference between both neuron types after cue presentation (first $100 \mathrm{~ms}$ ). (b) Neuronal activity in response to presentation fruit- and bacon-flavored rewards in putative dopamine (green) and non-dopamine neurons (blue), 300-400 ms after cue onset. All data represented as normalized z-scores. *Significant difference at $P<0.05$. Shaded gray area represents the reward-delivery interval.

of DA neurons, but not in other recorded neurons (Figure 3b). These results thus show class-specific neuronal activity that corroborate known properties of DA neurons.

Food restriction is necessary for cue-induced firing of DA neurons To assess the influence of feeding on reward processing, we measured midbrain VTA neuronal activity under conditions of short food restriction or ad lib access to chow.

Surprisingly, when measured under ad lib-fed conditions neither DA nor non-DA neurons show increased firing activity in response to cue or reward presentation (Figure 4a). Analysis of variance (repeated-measures ANOVA) revealed that there was no effect of time, group or interaction ( $F>0.592, P>0.378$ ) in salinetreated animals. Similarly, when all neurons were included in the analysis, no significant effect of time was observed $(F=1.010$, $P=0.426$ ). Together, these data thus indicate that under ad libitum access to food and water, DA neurons show minimal responsivity to reward predicting cues.

Pre-treatment with the anorexic hormone leptin suppresses food restriction-induced firing of DA neurons

To examine the effect of feeding hormones on cue-induced activity of DA neurons, we analyzed whether administration of ghrelin and leptin modulated this activity.

Leptin administration before recording sessions significantly decreased cue-induced neuronal activity in DA neurons measured under conditions of food-restriction. A repeated-measures comparison between saline and leptin-pretreatment conditions revealed a significant effect of time (Figure $4 \mathrm{~b}, \mathrm{~F}=4.076, P=0.001$ ) and a group $\times$ time interaction $(F=3.467, P=0.002)$. Post hoc analysis furthermore indicated a significant difference between groups following cue presentation $(t=4.233, P=0.000)$. In contrast, ghrelin treatment did not affect cue responding compared with saline-treated animals (Figure 4c, $F \leqslant 1.123$, $P \geqslant 0.353$ ). In ad lib-fed animals leptin or ghrelin administration did not alter event-related firing of DA neurons.

Overall we find that activity of midbrain DA neurons is increased during proximal cue presentation, in accordance with earlier findings. ${ }^{8}$ Importantly, this transient increase in event-related firing is only observed in animals that were food restricted and this increase is abolished by administration of leptin but not affected by ghrelin.

\section{DISCUSSION}

Here we show that a cue that predicts the availability of a food reward induces DA firing, but only in conditions of food restriction. Moreover, the anorexigenic hormone leptin can suppress this cue-induced neuronal activity. As such, these data provide a neural substrate through which metabolic information modulates encoding of cues associated with food reward on a millisecond timescale by DA neurons.

Neuronal activity measured in mildly fasted rats (that is, $2 \mathrm{~h}$ food restriction) revealed that while midbrain DA neurons strongly increase their activity to reward-predicting cues, both leptin pre-treatment and free access to chow before task execution abolished this cue-induced firing. Although ghrelin did affect baseline neuronal activity, no evidence was found for modulation of task-related activity of DA neurons.

Of particular relevance for these data is earlier work that showed that under anesthesia VTA DA neurons decrease their firing to intravenous leptin, but the functional consequences of leptin on DA signaling during behavior remained unclear. ${ }^{19}$ Importantly, as leptin affected neither reward preference, number of nosepokes, nor latency to nosepoke, these data suggest that leptin acts directly on the signaling of reward information. The subset of neurons that we recorded from under these conditions thus shows sensitivity to food restriction and to leptin but surprisingly did not significantly decrease task performance.

It is well established that operant behavior that requires a low motivational effort, like the single nosepoke that was required in this task, is resistant to accumbal DA lesions, ${ }^{7,34,35}$ similar to feeding behavior in a setting where food is freely available. ${ }^{36}$ Importantly, cue-induced accumbal DA release is seen during these tasks. ${ }^{3}$ This suggests that DA neuronal activity is present, but not required for basic task execution on a low-effort paradigm. Possibly DA activity, reflecting reward-prediction errors, affects task performance on a longer timescale. It is thus likely that the behavioral performance on this task is largely mediated by brain activity outside the VTA. Importantly, while leptin effects on behavior are seen on high motivational tasks like progressive ratio tasks, ${ }^{21}$ they are most pronounced on low effort feeding after multiple injections, or over multiple hours. ${ }^{37,38}$ Therefore, the elegance of this study is that we provide a task that is low in motivational load, but still induces DA burst firing. The reduction in firing we see by leptin therefore does not modify task performance, ruling out the possible confound that leptins effect on behavior is the cause for the changes in DA firing that we observe. 
a
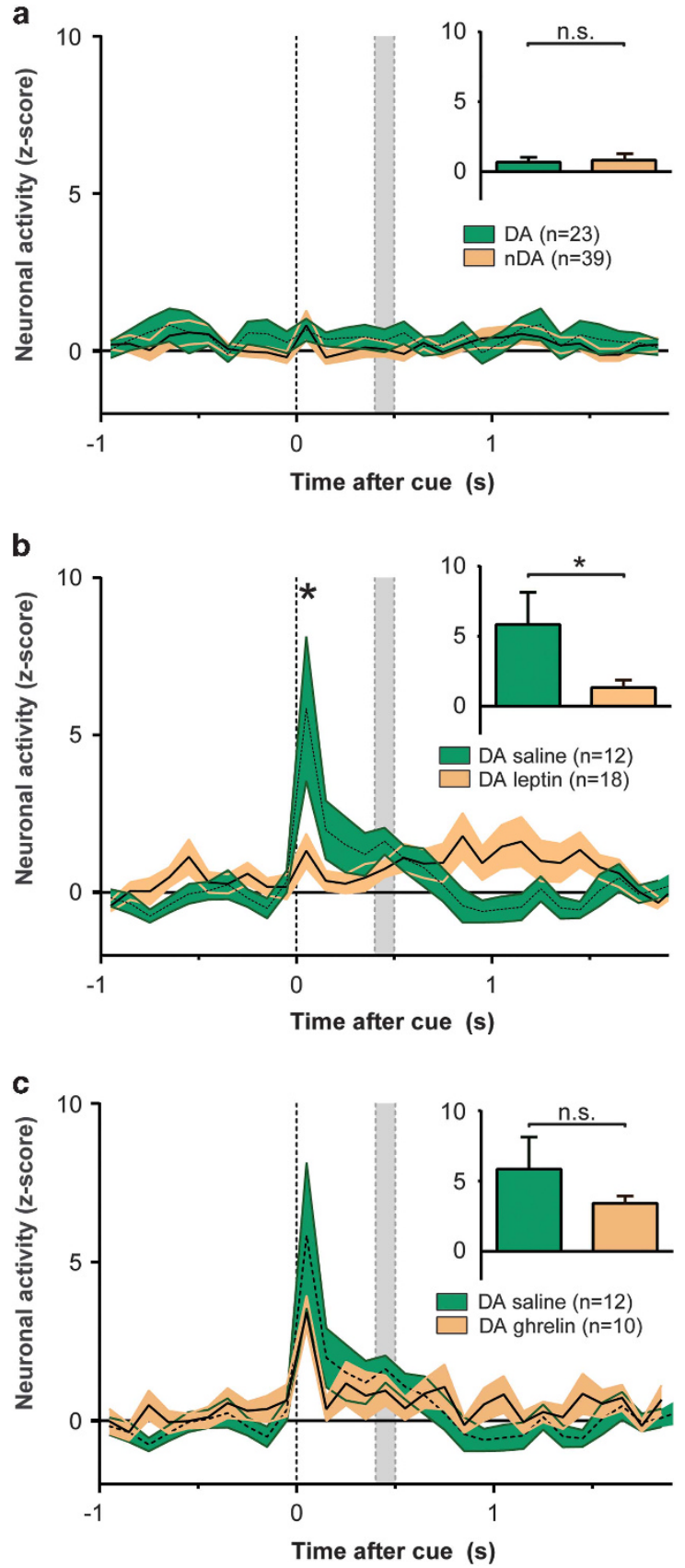

Figure 4. Leptin and access to food attenuates cue-induced firing. (a) The absence of cue-induced firing in dopamine (DA) and non-DA (nDA) neurons (resp. green and orange trace) following ad lib access to food. No significant difference was found between both neuron types after cue presentation (first $100 \mathrm{~ms}$; insert). (b) Leptin (orange trace) attenuates cue-induced firing of DA neurons in food-restricted animals (green trace). The insert shows a significant difference between both treatments after cue presentation (first $100 \mathrm{~ms}$ ), the control group is identical to the group shown in Figure $3 a$. (c) Ghrelin does not affect cue-induced firing in food-restricted animals (orange trace) compared with saline-treated animals (green trace). The insert shows the average firing activity for both conditions after cue presentation (first $100 \mathrm{~ms}$ ), the control group is identical to the group shown in Figure 3a. All data represented as normalized $z$-scores. *Significant difference $P<0.05$. Shaded gray area represents the reward-delivery interval.
Systemic treatment with the orexigenic hormone ghrelin decreased overall baseline activity of DA neurons under conditions of food restriction, but did not affect cue-induced activity. Such a dissociation between basal activity and burst firing has been reported before. Hyland et al., for instance showed that the average firing activity is not different between high and low bursting groups of neurons. ${ }^{30}$ In addition, modulation of excitatory inputs to VTA DA neurons affects burst firing, but not basal activity. ${ }^{39}$

Different effects of ghrelin on DA activity have been reported. Whereas in human subjects administration of ghrelin has been shown to increase activity in the midbrain, among other areas (for example, Malik et $a .^{26}$ ), reports from rodent studies are less consistent. Cone et al. ${ }^{40}$ previously showed ventricular, but not VTA, ghrelin injections increase accumbal DA release. Jerlhag et al. $^{20}$ found that intra-VTA ghrelin injections increase accumbal DA levels. These seemingly contradictory results are possibly due to the different routes of administration. As ghrelin receptors are expressed throughout brain and periphery, ${ }^{41}$ we chose the route of endogenous ghrelin by i.p. injection.

The recorded DA neurons show differential firing between a preferred and non-preferred reward, in line with earlier reports, ${ }^{9-12}$ and show burst patterns similar to those of DA neurons reported before. ${ }^{30,31}$ Although these data corroborate earlier work on the function of DA in the signaling of reward information, a need for (mild) food restriction to induce cue-induced firing has not been reported before. It should be noted that the majority of earlier work on DA signaling was done under conditions of food (or water) restriction. ${ }^{30,42,43}$ It has long been established that food restriction increases general activity and motivation, as well as the rewarding effect of lateral hypothalamic self-stimulation. ${ }^{15,44}$ More recently, it was found that food restriction changed basal activity of DA neurons, ${ }^{18}$ and here for the first time we show that it enhances reward processing by midbrain DA neurons.

The lateral hypothalamus sends dense projections to the VTA, containing a wide range of neuropeptides that modulate feeding behavior, including orexin/hypocretin, melanin-concentrating hormone and neurotensin. Interestingly, it has been reported that a subset of $\mathrm{LH}$ neurons that contain the leptin receptor project directly to the VTA, and might modulate DA activity. ${ }^{45}$ Although these LH leptin receptor containing neurons are presumed GABAergic, ${ }^{46}$ it has also been shown that leptin decreases glutamate-mediated excitatory inputs to DA neurons. ${ }^{47}$ Furthermore, receptors for leptin are also expressed on VTA DA neurons, enabling the possibility of a direct modulation. ${ }^{48}$ Taken together, it is likely that leptins effect on different brain structures contribute to downstream changes in DA signaling. Although we show that leptin and short food restriction modulate cue-induced firing of DA neurons, it remains to be investigated whether this is through a direct activation of receptors in the VTA, or whether inputs from other brain areas, like the $\mathrm{LH}$, are necessary for these effects.

DA neurons respond to reward-predicting cues with a transient increase in firing activity, enabling signaling of a reward-prediction error. ${ }^{8}$ Thus far, no studies have directly assessed the effects of feeding hormones on event-related firing of DA neurons in animals that are actively engaged in a reward-seeking task. The present data show that food restriction and leptin modulate DA signaling to cues associated with a food reward, without affecting task performance or actual feeding itself. Specifically, these data suggest that decreased reward value, through leptin administration, decreases cue-induced firing of midbrain DA neurons. In a similar manner, pre-feeding decreases neuronal responses to such reward-prediction cues. These findings confirm and extent previous reports on the effects of metabolic state and leptin treatment on the processing of food cues in humans. In these, leptin affected the response of reward-related neural 
structures upon presentation of food pictures without actual consumption. ${ }^{23-25}$ However, a direct modulation of DA activity by leptin, on a millisecond time-scale, was not shown before.

As such, these data provide the first evidence for a direct effect of metabolic signaling on the processing of reward-related information by midbrain DA neurons and provide a neuronal substrate of satiety-induced changes in motivated behavior. These data bring a new perspective to how leptin affects the midbrain DA system which is relevant to eating disorders and obesity.

\section{CONFLICT OF INTEREST}

The authors declare no conflict of interest.

\section{ACKNOWLEDGEMENTS}

The research leading to these results has received funding from the European Union's Seventh Framework Programme for research, technological development and demonstration under grant agreement $n^{\circ} 266408, n^{\circ} 245009, n^{\circ} 607310$, and the Netherlands Organization for Scientific Research (NWO/ALW Veni grant).

\section{REFERENCES}

1 Hebebrand J, Albayrak Ö, Adan R, Antel J, Dieguez C, de Jong J et al. 'Eating addiction', rather than 'food addiction', better captures addictive-like eating behavior. Neurosci Biobehav Rev 2014; 47: 295-306.

2 Gao Q, Horvath TL. Neurobiology of feeding and energy expenditure. Annu Rev Neurosci 2007; 30: 367-398.

3 Roitman MF, Stuber GD, Phillips PEM, Wightman RM, Carelli RM. Dopamine operates as a subsecond modulator of food seeking. J Neurosci 2004; 24: $1265-1271$.

4 Salamone JD, Correa M. Dopamine and food addiction: lexicon badly needed. Biol Psychiatry 2013; 73: e15-e24.

5 Stratford TR, Kelley AE. GABA in the Nucleus Accumbens Shell Participates in the Central Regulation of Feeding Behavior. J Neurosci 1997; 17: 4434-4440.

6 Van den Heuvel JK, Furman K, Gumbs MCR, Eggels L, Opland DM, Land BB et al. Neuropeptide $Y$ activity in the nucleus accumbens modulates feeding behavior and neuronal activity. Biol Psychiatry 2015; 77: 633-641.

7 Salamone JD, Correa M. Motivational views of reinforcement: implications for understanding the behavioral functions of nucleus accumbens dopamine. Behav Brain Res 2002; 137: 3-25.

8 Schultz W, Dayan P, Montague RP. A neural substrate of prediction and reward. Science 1997; 275: 1593-1599.

9 Roesch MR, Calu DJ, Schoenbaum G. Dopamine neurons encode the better option in rats deciding between differently delayed or sized rewards. Nat Neurosci 2007 10: $1615-1624$.

10 Cohen JY, Haesler S, Vong L, Lowell BB, Uchida N. Neuron-type-specific signals for reward and punishment in the ventral tegmental area. Nature 2012; 482: 85-88.

11 Fiorillo CD, Yun SR, Song MR. Diversity and homogeneity in responses of midbrain dopamine neurons. J Neurosci 2013; 33: 4693-4709.

12 Fiorillo CD, Tobler PN, Schultz W. Discrete coding of reward probability and uncertainty by dopamine neurons. Science 2003; 299: 1898-1902.

13 Van Zessen R, van der Plasse G, Adan RAH. Contribution of the mesolimbic dopamine system in mediating the effects of leptin and ghrelin on feeding. Proc Nutr Soc 2012; 71: 435-445.

14 Narayanan NS, Guarnieri DJ, DiLeone RJ. Metabolic hormones, dopamine circuits, and feeding. Front Neuroendocrinol 2010; 31: 104-112.

15 Fulton S, Woodside B, Shizgal P. Modulation of brain reward circuitry by leptin. Science 2000; 287: 125-128.

16 Hillebrand JJG, Koeners MP, de Rijke CE, Kas MJH, Adan RAH. Leptin treatment in activity-based anorexia. Biol Psychiatry 2005; 58: 165-171.

17 Pothos EN, Creese I, Hoebel BG. Restricted eating with weight loss selectively decreases extracellular dopamine in the nucleus accumbens and alters dopamine response to amphetamine, morphine, and food intake. J Neurosci 1995; 15 6640-6650.

18 Branch S, Goertz B, Sharpe A, Pierce J, Roy S, Ko D et al. Food restriction increases glutamate receptor-mediated burst firing of dopamine neurons. J Neurosci 2013 33: $13861-13872$

19 Hommel JD, Trinko R, Sears RM, Georgescu D, Liu Z-W, Gao X-B et al. Leptin receptor signaling in midbrain dopamine neurons regulates feeding. Neuron 2006; 51: 801-810.
20 Jerlhag E, Egecioglu E, Dickson SL, Douhan A, Svensson L, Engel JA. Ghrelin administration into tegmental areas stimulates locomotor activity and increases extracellular concentration of dopamine in the nucleus accumbens. Addict Biol 2007; 12: 6-16.

21 Figlewicz DP, Bennett JL, Naleid AM, Davis C, Grimm JW. Intraventricular insulin and leptin decrease sucrose self-administration in rats. Physiol Behav 2006; 89: 611-616.

22 Skibicka KP, Hansson C, Alvarez-Crespo M, Friberg PA, Dickson SL. Ghrelin directly targets the ventral tegmental area to increase food motivation. Neuroscience 2011; 180: 129-137.

23 Farooqi I, Bullmore E, Keogh J, Gillard J, O'Rahilly S, Flethcer P. Leptin regulates striatal regions and human eating behavior. Science 2007 317: 1355.

24 Frank S, Heni M, Moss A, Von Schnurbein J, Fritsche A, Häring HU et al. Leptin therapy in a congenital leptin-deficient patient leads to acute and long-term changes in homeostatic, reward, and food-related brain areas. J Clin Endocrinol Metab 2011; 96: 1283-1287.

25 Farr OM, Fiorenza C, Papageorgiou P, Brinkoetter M, Ziemke F, Koo B-B et al. Leptin therapy alters appetite and neural responses to food stimuli in brain areas of leptin-sensitive subjects without altering brain structure. J Clin Endocrinol Metab 2014; 99: E2529-E2538.

26 Malik S, McGlone F, Bedrossian D, Dagher A. Ghrelin modulates brain activity in areas that control appetitive behavior. Cell Metab 2008; 7: 400-409.

27 Sárvári M, Kocsis P, Deli L, Gajári D, Dávid S, Pozsgay Z et al. Ghrelin modulates the fMRI BOLD response of homeostatic and hedonic brain centers regulating energy balance in the rat. PLoS One 2014; 9: e97651.

28 Van der Plasse G, Merkestein M, Luijendijk MC, van der Roest M, Westenberg HG, Mulder $\mathrm{AB}$ et al. Food cues and ghrelin recruit the same neuronal circuitry. Int $J$ Obes (Lond) 2013; 37: 1012-1019.

29 Paxinos G, Watson C. The Rat Brain in Stereotaxic Coordinates. Sixth Edition. Acad Press, London 2006; 170: 547612

30 Hyland BI, Reynolds JNJ, Hay J, Perk CG, Miller R. Firing modes of midbrain dopamine cells in the freely moving rat. Neuroscience 2002; 114: 475-492.

31 Grace AA, Bunney BS. The control of firing pattern in nigral dopamine neurons: burst firing. J Neurosci 1984; 4: 2877-2890.

32 Abizaid A, Liu Z-W, Andrews ZB, Shanabrough M, Borok E, Elsworth JD et al. Ghrelin modulates the activity and synaptic input organization of midbrain dopamine neurons while promoting appetite. J Clin Invest 2006; 116 3229-3239.

33 Fiorillo CD, Song MR, Yun SR. Multiphasic temporal dynamics in responses of midbrain dopamine neurons to appetitive and aversive stimuli. J Neurosci 2013; 33: $4710-4725$.

34 Salamone J, Wisniecki a, Carlson B, Correa M. Nucleus accumbens dopamine depletions make animals highly sensitive to high fixed ratio requirements but do not impair primary food reinforcement. Neuroscience 2001; 105: 863-870.

35 Aberman JE, Salamone JD. Nucleus Accumbens dopamine depletions make rats more sensitive to high ratio requirements but do not impair primary food reinforcement. Neuroscience 1999; 92: 545-552.

36 Salamone JD. Different effects of haloperidol and extinction on instrumental behaviours. Psychopharmacology (Berl) 1986; 88: 18-23.

37 Halaas JL, Boozer C, Blair-West J, Fidahusein N, Denton D, Friedman JM et al. Physiological response to long-term peripheral and central leptin infusion in lean and obese mice. Proc Natl Acad Sci USA 1997; 94: 8878-8883.

38 Halaas JL, Gajiwala KS, Maffei M, Cohen SL, Chait BT, Rabinowitz D et al. Weightreducing effects of the plasma protein encoded by the obese gene. Science 1995; 269: 543-546.

39 Floresco SB, West AR, Ash B, Moore H, Grace Aa. Afferent modulation of dopamine neuron firing differentially regulates tonic and phasic dopamine transmission. Nat Neurosci 2003; 6: 968-973.

40 Cone JJ, McCutcheon JE, Roitman MF. Ghrelin acts as an interface between physiological state and phasic dopamine signaling. J Neurosci 2014; 34: 4905-4913.

41 Guan X, Yu H, Palyha O, McKee K, Feighner S, Sirinathsinghji D et al. Distribution of mRNA encoding the growth hormone secretagogue receptor in brain and peripheral tissues. Mol Brain Res 1997; 48: 23-29.

42 Schultz W, Apicella P, Ljungberg T. Responses of monkey dopamine neurons to reward and conditioned stimuli during successive steps of learning a delayed response task. J Neurosci 1993; 13: 900-913.

43 Ljungberg T, Apicella P, Schultz W. Responses of monkey dopamine neurons during learning of behavioral reactions. I Neurophysiol 1992; 67: 145-163.

44 Margules D, Olds J. Identical 'feeding' and 'rewarding' systems in the lateral hypothalamus of rats. Science 1962; 135: 374-375. 
45 Leinninger GM, Opland DM, Jo YH, Faouzi M, Christensen L, Cappellucci LA et al. Leptin action via neurotensin neurons controls orexin, the mesolimbic dopamine system and energy balance. Cell Metab 2011; 14: 313-323.

46 Leinninger GM, Jo Y-H, Leshan RL, Louis GW, Yang H, Barrera JG et al. Leptin acts via leptin receptor-expressing lateral hypothalamic neurons to modulate the mesolimbic dopamine system and suppress feeding. Cell Metab 2009; 10: 89-98.

47 Thompson JL, Borgland SL. Presynaptic leptin action suppresses excitatory synaptic transmission onto ventral tegmental area dopamine neurons. Biol Psychiatry 2013; 73: 860-868.
48 Figlewicz DP, Evans SB, Murphy J, Hoen M, Baskin DG. Expression of receptors for insulin and leptin in the ventral tegmental area/substantia nigra (VTA/SN) of the rat. Brain Res 2003; 964: 107-115.

(i) This work is licensed under a Creative Commons Attribution 4.0 International License. The images or other third party material in this article are included in the article's Creative Commons license, unless indicated otherwise in the credit line; if the material is not included under the Creative Commons license, users will need to obtain permission from the license holder to reproduce the material. To view a copy of this license, visit http://creativecommons.org/licenses/by/4.0/ 